\title{
Metachronous Polycythemia Vera and Multiple Myeloma in an Ethiopian patient
}

\section{Zablon Mesfin Anbessie ( $\nabla$ zabalon22@hotmail.com )}

Bethzatha General Hospital

Amha Gebremedhin GebreEgziabher

Tikur Anbessa Hospital: Black Lion Hospital

\section{Research Article}

Keywords: Polycythemia, Myeloma, Metachronous, Hematologic malignancy

Posted Date: January 19th, 2022

DOI: https://doi.org/10.21203/rs.3.rs-1260409/v1

License: (c) (i) This work is licensed under a Creative Commons Attribution 4.0 International License. Read Full License 


\section{Abstract}

Introduction- Dual hematologic malignancies whether synchronous or metachronous are a rare finding in the hematologic literature. A retrospective review has estimated $1.5 \%$ of patients with a single hematologic malignancy will go on to develop a second hematologic malignancy. The occurrence of polycythemia vera with multiple myeloma is an even rarer finding with only 18 cases being described over the past 75 years.

Case-A 52 years old male patient who was a known Jak-2 positive polycythemia vera patient on hydroxyurea and with reasonable hematologic profiles, insidiously developed severe anemia requiring transfusion, despite discontinuation of hydroxyurea. Along with the anemia the patient progressively developed fatigue, leg swelling, back pain, decreased urine output, and his creatinine increased from a baseline of $1.4 \mathrm{mg} / \mathrm{dl}$ to $9 \mathrm{mg} / \mathrm{dl}$. His examination was significant for massive hepatosplenomegaly. His labs showed lytic skull bone lesions, an IgG Lambda monoclonal spike, serum calcium of $13.3 \mathrm{mg} / \mathrm{dl}$, and a 24-hour urine protein of $7 \mathrm{~g}$.

Discussion and conclusion-We were able to demonstrate that our patient had a similar sociodemographic resemblance with prior reported cases but differed in that he had more aggressive myeloma. We advocate clinicians to have a high index of suspicion as well as an open mind for the consideration of dual hematologic malignancies during their practice.

\section{Introduction}

Dual hematologic malignancies whether synchronous or metachronous are such a rare occurrence in medicine that it is believed they occur coincidentally. They are usually diagnosed when clinical and laboratory finding discordance occurs in a patient, or, a patient is incidentally diagnosed during routine workup. It has been estimated that approximately $1.5 \%$ of patients with a hematologic malignancy will develop another hematologic malignancy. The distribution of simultaneous dual hematologic malignancies identified in the literature was; myeloid/lymohoid-50\%, lymphoid/lymphoid- $43 \%$, and myeloid/myeloid-7\%. The coexistence of Chronic lymphocytic leukemia (CLL) with Myeloproliferative neoplasm (MPN) is the most commonly described dual hematologic malignancy to date. ${ }^{1}$

The occurrence of polycythemia vera, an MPN characterized by myeloid cell clonal proliferation with different degrees of maturity and hematopoietic efficiency, preceding or along with multiple myeloma, neoplastic proliferation of plasma cells in the bone marrow that produce monoclonal immunoglobulins, is very rare. Polycythemia associated with myeloma has only been described in 27 cases over the past 75 years. Among the 27 cases, 18 had polycythemia vera with multiple myeloma, three had secondary polycythemia with myeloma, and six had unexplained polycythemia with multiple myeloma. ${ }^{2-5}$

Most of the cases described in the literature had been diagnosed with polycythemia vera based on red blood cell mass with no molecular studies. Most of the studies were also done before 1990 where therapy with radioactive phosphorous and local radiation was common for polycythemia vera. Based on these 
cases, the possible relationship, even though unclear has been hypothesized to be among clonal evolution, therapy-related secondary malignancy, the reversion of pluripotent stem cells to multipotent stem cells that become malignant, or coincidence. ${ }^{6-8}$

Since there have only been a handful of cases of dual hematologic malignancies to base prognostic and therapeutic approaches on, it is very difficult to make any strong recommendations. But, it has been suggested that the majority of simultaneous dual hematologic malignancies can be managed expectantly, and if both malignancies require treatment, treatment should be directed against the aggressive malignancy. Specific evidence regards polycythemia vera and plasma cell dyscrasias suggest that the course of polycythemia vera was not altered by plasma cell neoplasms and the use of hydroxyurea $>1000 \mathrm{mg} /$ day decreased the level of monoclonal (M)-protein in all subtypes of monoclonal gammopathies. ${ }^{1}$

To the Authors Knowledge, our patient is the 19th patient in the literature and the first patient from the African continent to be described with polycythemia vera and multiple myeloma. We hope our patient's case will add to the knowledge base of the cooccurrence of polycythemia vera and myeloma, and be a baseline for comparison, in the African continent.

\section{Case Report}

A 52 years old male patient presented to our emergency department being a known Janus Kinase-2 (Jak2) positive Polycythemia Vera patient for the past 6years on intermittent phlebotomy, hydroxyurea, and aspirin. He had been diagnosed with chronic portal vein thrombosis and cavernous transformation with esophageal varices at the same time and was on surveillance. He had a stable blood count on follow-up with a WBC of $13,000-24,000$ cells $/ \mathrm{mm}^{3}$, Hemoglobin of $13-16 \mathrm{gram} / \mathrm{dl}$, and a platelet count of 130,000 400,000 cells $/ \mathrm{mm}^{3}$ while taking hydroxyurea.

One month before presentation he developed progressive anemia to hemoglobin of $6 \mathrm{~g} / \mathrm{dl}$, which did not respond to discontinuation of Hydroxyurea and repeated blood transfusions. Two weeks before his presentation he started complaining of back pain with cola-colored urine and a progressive decline in his urine output. His serum creatinine which was normal at first but was checked to be $1.4 \mathrm{mg} / \mathrm{dl}$ during these symptoms progressively increased to a level of $9 \mathrm{mg} / \mathrm{dl}$ five days before his presentation. He was sent to the emergency department for a progressive decline in urine output with fatigue, intermittent vomiting, and leg swelling.

On examination, he looked lean with hyperpigmented facial skin. He had a blood pressure of $100 / 50 \mathrm{mmHg}$, a pulse rate of 81 beats/minute, a respiratory rate of 24 breaths/minute, a saturation of $96 \%$ on room air, and a temperature of $37^{\circ} \mathrm{C}$. He had an injected conjunctiva with left posterior basal onethird crepitation, his liver was enlarged with a smooth surface and sharp edge up to $6 \mathrm{~cm}$. below the right costal margin and the spleen was palpable $12 \mathrm{~cm}$. along its line of growth. 
His investigations confirmed multiple myeloma with hypercalcemia and acute kidney injury (Table-1, Figure-1-3).

He was admitted to the ward and he was initiated on intermittent hemodialysis and intravenous dexamethasone until he was stabilized. Subsequently, he was started on a Bortezomib, Cyclophosphamide, and Dexamethasone regimen (CyBorD). He was discharged three weeks later, improved with a urine output of One liter per day and a normal serum calcium level.

\section{Discussion}

We have demonstrated in our case report that unusual occurrence of myeloid and lymphoid malignancies, specifically polycythemia vera and multiple myeloma, is possible, and despite their occurrence being very rare, the diagnosis should be considered in any patient with a hematologic malignancy that develops atypical clinical, laboratory or therapeutic manifestations, unexpected from the primary malignancy. We were also able to show that the more aggressive malignancy should be given precedence in the management.

Based on a compilation of 15 cases of polycythemia with multiple myeloma, the mean age on presentation was 61 years (41-83) with $46 \%$ of the patients being males. One-third of the patients had the diagnosis of polycythemia and myeloma at the same time, while in the rest the diagnosis of polycythemia was made at a median of 4years ( 8 months to 20 years) before the diagnosis of myeloma. Nearly half of the patients had taken radioactive phosphorus for polycythemia treatment before the diagnosis of myeloma was made. ${ }^{7}$ These findings are similar to the remaining case reports not described in the compilation. Our patient is similar in most aspects to the above case reports but differs in that he has not taken any radioactive phosphorus. ${ }^{4,6}$

Most of the patients that had serum protein electrophoresis reported, described IgA monoclonal spikes. Most of the patients described also did not meet any of the CRAB criteria and there were no reports of dialysis requiring acute kidney injury. Our patient in contrast had IgG monoclonal spike with all of the CRAB criteria. Whether this difference occurred by chance, ethnicity, or presence of JAK-2 positivity is difficult to conclude. ${ }^{4,6,7}$ Most of the case reports did not describe the use of hydroxyurea and provided Melphalan, radiation, procarbazine, or steroids for treatment of their patients. ${ }^{7}$ While one case report had reported a partial response of the multiple myeloma with CyBorD treatment. Since it is too early to assess our patient's response to the myeloma therapy provided, it would be difficult to make any comparisons in this aspect. ${ }^{5}$

Despite the rare occurrence of two hematologic malignancies in the same patient we advise considering the diagnosis in patients who have atypical clinical and therapeutic features for the primary hematologic malignancy.

\section{Declarations}




\section{Ethics approval and consent to participate}

The patient had given written informed consent for the publication of the case report

\section{Consent for publication}

The patient had given written informed consent for the publication of the case report

\section{Availability of data and materials}

All have been included in the manuscript

\section{Competing interests}

None

\section{Funding}

None

\section{Authors contribution}

Zablon Mesfin- Diagnosis, treatment, followup of the patient, write up of the report, crossponding Author Amaha Gebremedhin- Diagnosis, treatment, followup of the patient,review of the report

\section{Acknowlegments}

Not applicable

\section{References}

1. Kotchetkov R, Ellison E, McLean J, Pressnail B, Nay D. Synchronous dual hematological malignancies: new or underreported entity? Hematology. 2018;23(9):596-9. doi:10.1080/10245332.2018.1466428.

2. Multiple myeloma: Clinical features, laboratory manifestations, and diagnosis - UpToDate. Accessed January 3. 2022. https://www.uptodate.com/contents/multiple-myeloma-clinical-features-laboratorymanifestations-and-diagnosis?search=multiple myeloma\&source=search_result\&selectedTitle=1 150\&usage_type=default\&display_rank=1.

3. Clinical manifestations and diagnosis of polycythemia vera - UpToDate. Accessed January 3. 2022. https://www.uptodate.com/contents/clinical-manifestations-and-diagnosis-of-polycythemia-vera? search=polycythemia vera\&source=search_result\&selectedTitle=1 110\&usage_type=default\&display_rank=1. 
4. Barange M, Subramanian PG, Chatterjee G, Patkar N, Gujral S, Tembhare P. Synchronous Presentation of Smoldering Multiple Myeloma (SMM) and Polycythemia Vera (PV)-A Rare Case Report. Clin Lymphoma Myeloma Leuk. 2017;17(1):e151. doi:10.1016/j.clml.2017.03.272.

5. Hutchison EJ, Taverna JA, Yu Q, Yeager AM. Polycythaemia: an unusual presentation of multiple myeloma. BMJ Case Rep. 2016;2016:1-4. doi:10.1136/bcr-2016-216686.

6. Inase N, Shichiri M, Marumo F. Secondary Polycythemia Associated with Multiple Myeloma. Jpn J Med. 1989;28(3):396-8. doi:10.2169/internalmedicine1962.28.396.

7. KOICHI MAEDA MD AND JOSEPH ABRAHAM MD. ill T8r. 2018;8(4).

8. Brody JI, Beizer LH, Schwartz S. Multiple myeloma and the myeloproliferative syndromes. Am J Med. 1964;36(2):315-9. doi:10.1016/0002-9343(64)90094-4.

\section{Table 1}

Table-1 Investigation summary 
Investigation

Date

\begin{tabular}{|c|c|c|c|}
\hline & $30 / 11 / 21$ & $3 / 12 / 21$ & $7 / 12 / 21$ \\
\hline WBC $\left(x 10^{3}\right.$ cells $\left./ \mathrm{mm}^{3}\right)$ & 21.5 & 33.5 & 32 \\
\hline Neutrophil (\%) & 79 & 76 & 92 \\
\hline Lymphocyte (\%) & 10 & 15 & 5 \\
\hline Monocyte (\%) & 10 & 9 & 3 \\
\hline Hemoglobin $(g / L)$ & 7 & 6 & 8 \\
\hline Hematocrit (\%) & 22 & 18 & 24 \\
\hline Platelet $\left(\times 10^{3}\right.$ cells $\left./ \mathrm{mm}^{3}\right)$ & 200 & 188 & 109 \\
\hline Creatinine (mg/dl) & 9 & - & - \\
\hline Albumin (g/dl) & 1.6 & - & - \\
\hline Total protein (g/dl) & 12 & - & - \\
\hline Calcium (mg/dl) & 11.4 & 8.2 & 7.9 \\
\hline Corrected calcium (mg/dl) & 13.3 & 10.1 & 9.8 \\
\hline Potassium (meq/L) & 5.5 & 5.9 & 4.2 \\
\hline Sodium (meq/L) & 126 & 115 & 130 \\
\hline Phosphate (mg/dl) & 5.6 & 6.1 & 4.1 \\
\hline Compliment-3 (C3) (mg/dl) & - & $20.9(90-170)$ & - \\
\hline Compliment-4 (C4) (mg/dl) & - & $<8(10-40)$ & - \\
\hline
\end{tabular}

Thoracolumbar MRI-compression fracture of the vertebral body of T8

Echocardiography-Left atrium and right-side cardiac chambers are dilated with an ejection fraction of $60 \%$, and a peak pulmonary pressure gradient of $62 \mathrm{mmHg}$, with grade II diastolic dysfunction.

Abdominal ultrasound- Normal sized kidneys, cavernous transformation of the portal vein with massive hepatosplenomegaly and cholelithiasis,

Bone marrow aspirate and biopsy- Anisocytosis of red blood cells with plasma cells in the periphery with $<1 \%$ being counted. The bone marrow is cellular $(80 \%)$ with $25 \%$ of the cells being plasma cells.

JAK-2 V617F mutation- Positive

Serum EPO levcel-1 milliunit per liter

\section{Figures}


Figure 1

Serum protein electrophoresis and immunofixation with serum-free light chain assay

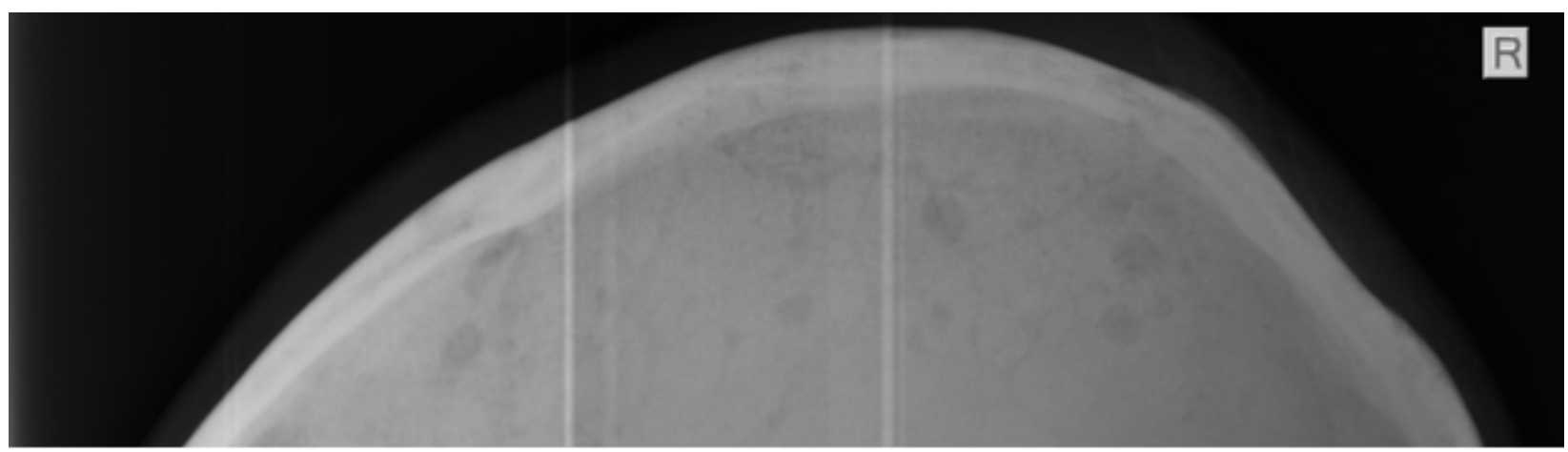

Figure 2

Skull x-ray showing lytic lesions

2

Figure 3

Bone marrow Biopsy showing cellular marrow with plasma cells 